\title{
Relative Humidity Prediction Model with Missing Data Refinement Using a Long Short-Term Memory Neural Network
}

\author{
Inyoung Park, Jiwon Lee, Hyun Soo Kim, Chul Han Song, Hong Kook Kim \\ Gwangju Institute of Science and Technology \\ 123 Cheomdangwagiro, Bukgu, Gwangju 61005, South Korea \\ pinyoung@gist.ac.kr, easy-one@gist.ac.kr, hskim@gist.ac.kr, chsong@gist.ac.kr, hongkook@gist.ac.kr
}

\section{Extended Abstract}

Climate change has been a global challenge that has depended primarily on model-based methods. Nowadays, global warming not only intimidates humankind but also threatens the ecosystem due to its unpredictability. Among various pollutant factors, aerosol is considered as a major factor for climate change because it is known to affect global warming [1]. If aerosol can be predictable, we can expect to prepare for abnormal weather. Due to prediction accuracy should be reliable, there is work has high priority. As is known, relative humidity (RH) affects to density of aerosol [2]. To build an aerosol prediction model, RH data are indispensable but there is a limitation in collecting data that are always detectless.

In this paper, an RH prediction model is proposed which is based on a deep neural network by using real observed weather data from multiple locations. In particular, a long short-term memory (LSTM) neural network, which is a type of recurrent neural network (RNN), is utilized because LSTM is known as suitable for a very long period of data [3, 4, 5]. Furthermore, different types of LSTMs are compared to characterize the time-series traits of climate data. Moreover, in order to include climate data even when the RH is missing, the proposed TH prediction model first fills missing data of RH with their predicted ones by using LSTM.

To evaluate the performance of the proposed LSTM-based RH prediction model, the LSTM is trained by using 6 years climate data (from August 2011 to December 2016) from 11 different locations of South Korea, including hourly-based measurements for temperature, relative humidity, wind speed, wind direction, precipitation and accumulated prediction. The performance of the proposed LSTM-based RH prediction model is measured in terms of the root-mean-squared error (RMSE) between the actual RH and its predicted one. First, the proposed method is applied to predict RH for 1 hour. Consequently, average RMSE of 4.71 is obtained. Next, the proposed method is trained to predict the RH for next 12 hours by taking previous 12 hours of climate factors as the input. As a result, average RMSE of 8.19 is achieved when the defected RH is ignored for training the LSTM. On the other hand, the missing RH data are filled by the proposed method, RMSE is reduced into 7.90 .

So far, we have presented an RH prediction model that keeps track short term for the climate change period by taking into account the major influence of the primary weather variable. Although the proposed model refines missing RH data here, it can be applied to any missing data set. Furthermore, the prediction for long-term time steps of multiple locations with various weather data would make it possible to extend the proposed RH prediction model with refinement of missing climate factors.

\section{Acknowledgement}

This research was supported by the National Strategic Project-Fine particle of the National Research Foundation of Korea(NRF) funded by the Ministry of Science and ICT(MSIT), the Ministry of Environment(ME), and the Ministry of Health and Welfare(MOHW).(NRF-2017M3D8A1092022) 


\section{References}

[1] A. J. McMichael, R. E. Woodruff, and S. Hales, "Climate change and human health: present and future risks," The Lancet, vol. 367, no 9513, pp. 859-69, 2006.

[2] M. Schaap, A. Apituley, R. M. A. Timmermans, R. B. A. Koelemeijer, and G. de Leeuw, "Exploring the relation between aerosol optical depth and PM2.5 at Cabauw, the Netherlands," Atmospheric Chemistry and Physics, vol. 9, pp. 909-925, 2009.

[3] Q. Cao, B. T. Ewing, M. A. Thompson, "Forecasting wind speed with recurrent neural networks," Eur. J. Oper. Res. vol. 221, no. 1, pp. 148-54, 2012.

[4] I. Maqsood, M. R. Khan, and A. Abraham, "An ensemble of neural networks for weather forecasting," Neural Computing \& Applications, vol. 13, no.2, pp. 112-122, 2004.

[5] I. Park, J. Lee, B. Kim, J. H. Kim, and H. K. Kim, "Climate change prediction based a long short-term memory neural network," in Proc. Of 4th World Conference on Climate Change, vol. 8, pp. 119, 2017. 